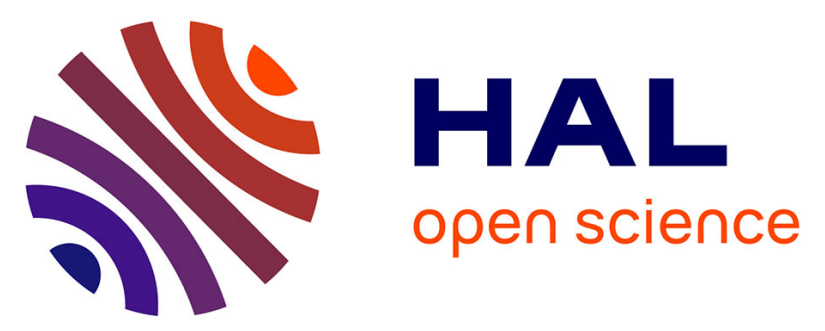

\title{
Chemotherapy versus tamoxifen versus chemotherapy plus tamoxifen in node-positive, oestrogen receptor-positive breast cancer patients. Very late results of the 'gruppo di ricerca per la chemio-ormonoterapia adiuvante (GROCTA)' 01-Trial in early breast cancer
}

Francesco Boccardo, Pamela Guglielmini, Andrea Parodi, Alessandra Rubagotti

\section{To cite this version:}

Francesco Boccardo, Pamela Guglielmini, Andrea Parodi, Alessandra Rubagotti. Chemotherapy versus tamoxifen versus chemotherapy plus tamoxifen in node-positive, oestrogen receptor-positive breast cancer patients. Very late results of the 'gruppo di ricerca per la chemio-ormonoterapia adiuvante (GROCTA)' 01-Trial in early breast cancer. Breast Cancer Research and Treatment, 2011, 126 (3), pp.653-661. 10.1007/s10549-011-1405-6 . hal-00616696

\section{HAL Id: hal-00616696 https://hal.science/hal-00616696}

Submitted on 24 Aug 2011

HAL is a multi-disciplinary open access archive for the deposit and dissemination of scientific research documents, whether they are published or not. The documents may come from teaching and research institutions in France or abroad, or from public or private research centers.
L'archive ouverte pluridisciplinaire HAL, est destinée au dépôt et à la diffusion de documents scientifiques de niveau recherche, publiés ou non, émanant des établissements d'enseignement et de recherche français ou étrangers, des laboratoires publics ou privés. 
Chemotherapy versus Tamoxifen versus Chemotherapy plus Tamoxifen in Node-positive, Estrogen Receptorpositive breast cancer patients. Very late results of the "Gruppo di Ricerca per la Chemio-Ormonoterapia Adiuvante (GROCTA)" 01-Trial in early breast cancer.

Francesco Boccardo ${ }^{* 1,2}$, Pamela Guglielmini ${ }^{1,2}$, Andrea Parodi ${ }^{3}$ and Alessandra Rubagotti ${ }^{1,2}$ on behalf of the participants in the GROCTA 01 Trial $^{4}$

${ }^{1}$ Department of Oncology, Biology and Genetics, University of Genoa; ${ }^{2}$ Department of Medical Oncology, National Cancer Research Institute, Genoa; ${ }^{3}$ Department of Biostatistics, University "La Bicocca", Milan, Italy . ${ }^{4}$ See list reported in Acknowledgements.

*Corresponding Author: Prof. Francesco Boccardo, MD, National Cancer Research Institute and the University of Genoa, Largo Rosanna Benzi 10, 16132 Genoa, Italy. e-mail: fboccardo@unige.it. Phone number: +390105600560 Fax number: +39010352753

Running head: Chemotherapy vs Tamoxifen vs Chemotherapy + Tamoxifen in early breast cancer.

Presented in part at the ASCO Annual Meeting,Chicago, USA, June 4-8, 2010 


\section{ABSTRACT}

Background and study purposes: firstly, to evaluate whether the benefits of combined chemotherapy (CT) and Tamoxifen (T), previously documented in the GROCTA-01 Trial, were long-lasting and, secondly, to show whether ER or PgR levels could allow the identification of patients who could benefit from $\mathrm{T}$ alone.

Patients and methods: 504 node-positive, ER-positive, women were randomly assigned to $10 \mathrm{CT}$ courses or to 5 years of $\mathrm{T}$ or to the combination of the two (CTT). Disease-free (DFS) and overall survival (OS) were the primary trialendpoints. DFS data were updated in $75 \%$ of patients and S data in $95 \%$ of them. Cox regression models were used to check for prognostic features, to estimate Hazard Ratios (HRs) for treatment comparisons and to test for possible interaction between variables and treatment effects. Interactions between treatments and ER or PgR median levels were studied with the Sub-population Treatment Effect Pattern Plot (STEPP) methodology.

Results: after a median follow-up time of 21 years, the DFS and OS benefits, previously favouring T over CT, continued to be observed, even though they were more evident in the first 6-7 years. The CTT advantages of DFS and OS over $\mathrm{T}$ alone were also confirmed. However, the additional benefit was limited to the first 10-12 years as $\mathrm{S}$ curves crossed-over afterwards. After STEPP analysis, neither ER or PgR concentrations fully discriminated the patients who could benefit from $\mathrm{T}$ alone.

Conclusions: even after such a long follow-up time, we have demonstrated that $\mathrm{T}$ is an effective alternative to CT for node-positive, ER-positive, breast cancer patients, regardless of their actual menopausal status, and that the additional benefit, especially on late survival, provided by the addition of $\mathrm{CT}$ to this anti-oestrogen, was minimal.

KEYWORDS: early breast cancer, chemotherapy, Tamoxifen, chemotherapy plus Tamoxifen 


\section{Introduction}

The combination of chemotherapy (CT) together with Tamoxifen (T), or aromatase inhibitors in post menopause, is the present paradigm to manage node-positive, ER-positive early breast cancer patients [1], though there is increasing evidence that endocrine manipulations alone might possibly represent an appropriate treatment for selected groups of these women [2-4].

Several individual trials and large meta-analysis have contributed to the building-up of the present state of art [5-12]. The GROCTA 01-Trial is one of the trials which concurred to this task. In fact, the original trial aim was to investigate whether in ER-positive women, T might represent a valuable alternative to CT (the standard at the time the trial was designed) and whether the co-administration of the two would provide additional benefits. Herein, we report the 25-year results of our trial with two major aims: 1) to show the long-term outcomes, both overall and in pre-defined groups, in order to evaluate if early benefits achieved by CTT persisted, also after such a prolonged follow-up time; and- 2) to show treatment outcomes according to quantitative ER or PgR levels in order to assess whether it could be possible to define, though on a retrospective basis, the preferable therapeutic choice according to tumor-receptor concentrations.

\section{Methods}

\section{Patients and procedures}

The GROCTA 01 Trial was a phase 3, open-label, parallel-group randomised controlled trial.

Details about study design, assigned treatments and main results have been previously published [6,13]. Five hundred and four, 35-65 year old patients with node-positive, ER-positive tumors and no evidence of distant metastases were recruited. Patients were well-balanced according to median age at entry, menopausal status, treatment of primary (either radical mastectomy or segmental mastectomy followed by breast irradiation), tumor size and number of involved nodes. Within 6 weeks from mastectomy, patients were randomly allocated to ( according to a 1:1:1 ratio), and administered one of the three following treatments: (1) T: $10 \mathrm{mg}$ orally three times per day for 5 years or until disease relapse; (2) combination chemotherapy (CT) with the following regimens: cyclophosphamide $500 \mathrm{mg} / \mathrm{m}^{2}$ plus methotrexate 40 $\mathrm{mg} / \mathrm{m}^{2}$ and fluorouracil $600 \mathrm{mg} / \mathrm{m}^{2}(\mathrm{CMF})$ for a total of 6 courses, followed by epirubicin $75 \mathrm{mg} / \mathrm{m}^{2}$ for another 4 courses. All drugs were given intravenously (IV) every 3 weeks. In the case of myelotoxicity occurring, drugs dosages were not reduced but treatment restart was usually delayed for 1 or 2 weeks until recovery; or-(3) a combination of both treatments (CTT) according to the same dosages and schedules. 
Patients were informed about the experimental nature of this trial which had been approved by the Human Investigations Committee of the National Cancer Research Institute of Genoa, Italy, in line with Italian 1 Legislation of that time.

\section{Hormonal receptor assay}

Tumor-ER concentration was measured using the standard dextran-coated charcoal technique and a cut-off value equal to 10 cytosol fmol/mg of protein was arbitrarily chosen to define the positivity of the assay and to select the patients who were suitable for recruiting into the trial. PgR assay was not mandatory, though it was performed almost in all patients according to the same technique. No entry restrictions were planned on the basis of $\operatorname{PgR}$ concentration.

\section{Trial end-points}

Disease-free survival (DFS), -defined as the time from randomization to local-regional recurrence, recurrence in the omo-lateral breast, distant metastases and contra lateral breast cancer, as well as overall survival (OS), defined as the time from randomization to any death, were the major trial end-points.

\section{Follow-up studies and data collection}

Patients were clinically examined every 3 months for the first 3 years, then every 4 months until the fifth year. A clinical check-up every 6 months was planned thereafter for a further 5 years. During the first five years, blood counts were repeated every 3 months, chest x-rays every 6 months and a yearly bone scan A mammography was also repeated every year. Chest tomography, liver scan and/or ultrasonography and all other investigations were carried out whenever deemed necessary by the patient's physician. Beyond the $10^{\text {th }}$ year, follow-up was continued at the discretion of local investigators or the patients' requirements, though commonly a mono- or bilateral mammogram was obtained on a yearly basis.

For the purposes of the present analysis, information about the patients' status was obtained from consulting the flow charts of the women still being followed up. The vital status of women who discontinued their attendance to the outpatients clinics for more than one year or who had not been able to have a follow-up in the more recent years, was checked directly by phone call or by consulting local tumour registries or the registry office of a patient's place of residence.

It was possible to update DFS data for $75 \%$ of patients and $\mathrm{S}$ data for $95 \%$ of them. 
Whenever possible, causes of death were recorded in detail. However, the cause of death was unknown for 45 patients. In these cases, we arbitrarily choose to include the deaths occurring after documented disease-relapse among breast cancer-related deaths while all other lethal events were defined as breast cancer-unrelated.

All data were collected through appropriate CRFs and centralized at the Clinical Trials Unit of the Coordinating Center.

\section{Sample size calculation}

On the basis of literature data available at the time the study was designed, a 5-year disease recurrence rate of 40-50\% was calculated for the patients assigned to CT. Because it was expected that the addition of Tamoxifen to chemotherapy would reduce this rate by about $20 \%$, it was estimated that for an alpha error $=5 \%$ and a beta error $=20 \%, 100$ patients per arm would provide the trial with an adequate statistical power to detect the superiority of CTT over CT under previous conditions. Due to it not being possible to reliably predict the outcome of $\mathrm{T}$ alone (as findings with $\mathrm{T}$ monotherapy available at that time were very preliminary and had been obtained from a mixed population of ERpositive and ER-negative patients), it was arbitrarily deemed that a comparable number of patients (i.e. 100) would be adequate to show non-inferiority of $\mathrm{T}$, in respect to $\mathrm{CT}$ alone. However, to be prudent, predicted recruitment was augmented by $50 \%$ for each arm.

\section{Statistical analysis}

DFS and S curves were constructed using the Kaplan-Meir method and treatment effect comparisons were obtained through the log-rank test [14]. Cox proportional hazards regression models [15] were used to check for prognostic features, to estimate Hazard Ratios (HRs) and 95\% confidence intervals (CIs) for treatment comparisons, as well as to test for interaction between variables and treatment effects. Menopausal status at trial entry, tumor ( $t$ ) size, hormone receptor levels and the number of involved nodes were included in the models. Hazard Rate curves for time to progression or death were smoothed by use of an Epanechinikov kernel with optimum bandwidth chosen by cross validation (STATA 9.0 sts graph command) [16]. Cumulative-incidence estimates for breast cancer-related and breast cancer-unrelated deaths were performed to check for competing risks. The difference between cumulative incidence curves in the presence of competing risks was tested using Fine and Gray's test [17].

Interactions between treatments and ER or PgR median levels (in fmol/mg of proteins) were studied by means of the non-parametric STEPP (Sub-population Treatment Effect Pattern Plot) methodology.

For this STEPP analysis, each sub-population contained approximately 100 patients and each subsequent subpopulation was formed by moving from left to right, dropping approximately 5 patients with the lowest values of ER or 
PgR and adding approximately 5 patients with the next higher values of ER or PgR (SAS 9.2). Treatment effects estimated within each ER or PgR sub-group level were measured in terms of HRs obtained from Cox models [18].

All probability values were obtained from two-sided tests. All statistical tests were carried-out using SPSS package (version 18.0), SAS (version 9.2), R(version 2.9.2) and STATASE10.

\section{Results}

Trial recruitment started on November 1, 1983 and was terminated on June 30,1987, having largely achieved the predicted size. In total, 510 patients were entered into the study. Six patients were excluded from the trial because they were entered by a Center that was withdrawn due to it not providing any additional patient information after randomization.

All the remaining 504 randomized patients ( $\mathrm{CT}=165 ; \mathrm{T}=168$; CTT 171) have been included into the analysis for the intention to treat method. The main characteristics of these patients are summarized in Table 1.

At a median follow-up time of 21 years (range: 0.5-26 years), -overall-, 316 patients developed a well-documented disease-related event and 352 died. Fig. 1 and 2 show DFS and S curves in all patients and in each menopausal subgroup. Even after such a long follow-up time, both T and CTT were confirmed to yield significantly better DFS results. However, the differences in respect to CT alone were more evident in postmenopausal women. In fact, there were no major differences between groups in pre-menopause. Comparably, $\mathrm{T}$ and CTT fared better than CT alone also relative to OS, while again no significant differences emerged between the two in this regard. The smoothed hazard estimates (Fig. 3) show, in addition, that the advantage favouring T, either alone or combined with CT, over CT alone, was quite exclusively confined to the first 6-7 years both in terms of DFS and of OS. No significant differences in the hazards of disease-progression or death were evident from Year 11 to Year 25.

CTT also confirmed to yield better DFS results than T alone, especially during the first 10-12 years and especially in post-menopausal women. However, there was no statistically significant difference between the two. Comparably, there was also no statistically significant difference between the two relative to OS. In fact, no difference at all between CTT and CT, even at 25 years of follow-up, was evident in menopausal women (Fig 2c). .Noteworthy is the fact that the S curves of the patients assigned to T or CTT crossed over between Year 10 and Year 12. In order to better investigate S curve cross-overs, competing risk analysis was performed according to causes of death as previously defined (Fig. 4). It is also worth noting that both cancer-related and cancer-unrelated deaths increased overtime. However, while there was no difference in breast cancer-unrelated mortality within the groups, breast cancer-related mortality risk was significantly higher for the patients initially assigned to $\mathrm{CT}$ and maintained so, even long term. 
Finally, there was no statistically significant difference in treatment interactions according to the number of involved nodes relative either to DFS or to OS (data not shown).

It should be noted that all outcome results provided have been adjusted for menopausal status at entry (pre vs. post), the number of involved nodes ( $1-3$ vs. $>3)$, t size $(<2 \mathrm{~cm}$ vs. $>2 \mathrm{~cm})$ and median PgR level $(<=55 \mathrm{vs} .>55 \mathrm{fmol} / \mathrm{mg})$. As shown in Table 2, all previous variables and assigned treatment were statistically significant predictors, both for the DFS (all variables except menopausal status) or for the OS (all variables) of the whole cohort of patients. In contrast to PgR levels, which were prognostic even when the positivity threshold was arbitrarily fixed at a value of $10 \mathrm{fmol} / \mathrm{mg}$ (similarly to ER), the ER levels were not prognostic at all, regardless of the cut-off level employed.

As previously described, non-parametric STEPP analysis was used to better define the putative value of ER or PgR quantitative levels in selecting the patients who might derive the greatest benefit from combining CT together T. As shown in Fig. 5a, which shows the STEPP data relative to DFS, the addition of CT to T appeared to benefit the patients with the lowest and the highest ER values, whilst providing no sustained benefit in the patients with intermediate values (between 40 and $100 \mathrm{fmol} / \mathrm{mg}$ ). However, differences between treatments were not statistically significant at any point, except for the sub-group of patients with a median ER value equal to $172 \mathrm{fmol} / \mathrm{mg}(\mathrm{p}<0.04)$. The situation was somewhat different relative to OS (Fig. 5b). In fact, the addition of CT to T appeared to produce a slight benefit only in women with lower ER or PgR values, while it did not show to produce any benefit at all in the women with ER or PgR values above 50-60 fmol/mg, though again differences were not statistically significant.

\section{Discussion}

Compared to present standards, our study has several limitations which result in the necessity of using caution before transferring the findings of the present analysis to today's clinical practice. Firstly, the study is to be considered undersized. We can say that 500 patients were a respectable number in 1983 when the trial was open to recruitment but, nowadays, this number qualifies the trial as a small one in respect to present day standards, though the predicted recruitment was largely achieved. The small size, in fact, renders the trial unpowered in respect to sub-group analysis. An additional objection might be that the CT regimen used by us is not likely to completely fit the canons of modern chemotherapy, even though it included an anthracycline. The choice to deliver T concurrently with CT might also be questionable according to present recommendations which suggest the use of $\mathrm{T}$ at the completion of CT [1]. However, this issue is still controversial, especially in respect to pre-menopausal women, and there is no definite proof as yet that sequencing CT with T might be more appropriate than giving the two concurrently [19,20]. In spite of its limitations, our trial has the strength of a very long follow-up and of adopting a "modern" design based on the use of an appropriate 
$\mathrm{T}$ scheduling (5 years) and of ER positivity as a criterion to prospectively select the patients suitable for the comparisons on study.

This long-term analysis clearly shows that the significant advantage of $\mathrm{T}$ over $\mathrm{CT}$ persisted throughout the years. However, the magnitude of the effect differed in the two menopausal sub-groups. In fact, there was no significant difference either in terms of DFS or of OS between CT and T among pre-menopausal women. Findings were different in post-menopausal women and, in fact, the patients assigned to $\mathrm{T}$ showed a huge advantage over those assigned to CT, which, once again, maintained itself over-time, up to the $25^{\text {th }}$ year, thus definitively confirming that endocrine therapy, namely T, should represent the mainstay to manage ER-positive post-menopausal women, regardless of the severity of nodal involvement. Moreover, the analysis of smoothed hazard estimates clearly indicates that the benefit achieved by $\mathrm{T}$ and CT, respectively, differ mainly during the first 6-7 years with, the risks maintaining the same degree in size thereafter, up to Year 25. There is fairly clear evidence now that ER-positive tumors might be less sensitive to adjuvant CT, while they are obviously expected to respond very well to endocrine manipulations [12,21]. Therefore, in spite of the well-documented carry-over effect both of $\mathrm{T}$ and of $\mathrm{CT}$ [12], which is also clearly demonstrated by our own present findings, it appears that the different chemo- and endocrine sensitivity of E- positive women might play a more relevant role in determining early, rather than late, outcomes.

Given that $\mathrm{T}$ is not inferior to $\mathrm{CT}$, rather it comes out as the favourable "winner" over CT from pair wise comparisons, even after such a long follow-up time, the question remains as to whether the addition of CT was able to further improve the results and whether the benefit observed is so relevant to identify the combination as the advisable standard for all node-positive women.

If we look at the results achieved by combined treatment in respect to DFS, we see that the addition of CT to T was able to induce a small advantage which resulted in being roughly the same size in the whole cohort of women and in each menopausal sub-group. This is strongly supportive of the fact that the lack of statistical significance in our trial is likely to be due to an effect of its small size. However, this additional benefit appears to be limited to the first years and to diminish over time. This trend also comes out from the analysis of smoothed hazard estimates. In fact, this analysis shows an advantage favouring the combined treatment only during the first years. This might reflect the putative effect of $\mathrm{CT}$ in controlling primary resistance to $\mathrm{T}$, a condition which has been described for selected groups of tumors also in the presence of ER or PgR positivity [22]. Relative to S, the tendency of curves towards crossing after a few years in the whole groups is likely to mainly reflect the tendency observed in pre-menopausal women. It is not so easy to explain the observed trend towards crossing. However, competing risk analysis of deaths appears to rule out that it might be related to the different incidence of breast cancer-unrelated deaths between groups. Once again, regardless of the mechanisms involved, it is a fact that, overall, the combination of CT with $\mathrm{T}$ did not improve the long term results 
achieved by $\mathrm{T}$ alone, especially in post-menopausal women, where the early DFS benefits achieved by the combination did not translate into a mortality advantage at any time.

In view of the limited benefit provided by the addition of CT to T in ER-positive patients, especially in post-menopause, it would be advisable to be able to discriminate a priori the women who might be safely managed by endocrine therapy alone. Through the STEPP analysis of our data, we tried to see whether quantitative ER or PgR values might help in discriminating such patients, especially in respect to their survival expectation. Indeed, the addition of CT to T did not appear to benefit the women with ER or PgR values above 50-60 fmol/mg at all. However, overall differences were not statistically significant. Thus ER or PgR quantitative levels do not appear to be completely affordable to indicate which women could be safely managed through $\mathrm{T}$ alone and additional bio-molecular predictors of endocrine responsiveness are probably required. The results reported on by Albain et al. [4] suggest that this might be the case. Albain's trial compared CAF vs. CAF plus T given concurrently vs. CAF followed by $\mathrm{T}$ in a population of node-positive, ER-positive post-menopausal women, demonstrating that the patient-subset with high ER concentration and HER-2 negative disease did not benefit further from CAF added to T. More recently, these investigators have reported on the predictive value of the 21-gene recurrence score (RS) assay originally described by Paik et al. [23] as being able to predict the benefit from CMF added to $\mathrm{T}$ in a group of ER-positive, node-negative women. In summary, no significant benefit, either in terms of DFS or of OS, was achieved by combining CAF with $\mathrm{T}$ in the low or intermediate RS sub-groups [24]. However, these investigators themselves conclude that prospective studies with larger samples sizes are essential to confirm the value of bio-molecular characterization and of multi-gene assays in establishing who benefits most from modern endocrine therapy and chemotherapy [24].

In conclusion, we have confirmed that, even after such a long follow-up time, $\mathrm{T}$ demonstrated to represent an effective alternative to CT for node-positive, ER-positive, breast cancer patients, regardless of their actual menopausal status, and that the additional benefit, especially on late survival, provided by adding $\mathrm{CT}$ to $\mathrm{T}$, was minimal. Thus, especially in post-menopausal women, the early benefits which might be expected from the addition of CT to T should be balanced with the low, if any, probability of a late mortality advantage.

\section{ACKNOWLEDGEMENTS}

The GROCTA 01 trial was originally funded in part by an unrestricted grant from ICI-Pharma (Milan, Italy). No funding was obtained by any entity to support present analysis.

The authors are indebted to M. Parodi and L. Zinoli (National Cancer Research Institute, Genoa,Italy) for their assistance in Data Management and to Dr C. Casella (Genoa Tumor Registry) for her valuable help in mortality data updating. 
They are also indebted to Ms Suzanne Patten for reviewing the language format.

Finally, they wish to express their gratitude to all the patients and the investigators who participated in this trial, namely, to the investigators listed below who have actively contributed to updating all data: M. Cantore (Ospedale Civile, Carrara); M. Mangoni (Ospedale Careggi, Firenze); C. Caroti (Ospedale Galliera, Genova); E. Rinaldi (Ospedale Civile, Magenta); E. Aitini (Ospedale Civile, Mantova); S. Banducci (Ospedale Civile, Merate); S. Girelli (Ospedale Fatebenefratelli, Milano); D. Aldigretti (Istituto Scientifico St Raffaele, Milano); B. Agostara, A. Traina (Ospedale Oncologico M. Ascoli, Palermo); G. Cruciani (Ospedale S. Maria delle Croci, Ravenna); C. Boni, R. Gnoni ( Ospedale S. Maria Nuova, Reggio Emilia); D. Guarneri ( Ospedale Civile, Sanremo); A. Farris, A. Lai (Oncologia Clinica, Università, Sassari); P.Sismondi, N.Biglia (I Clinica Ostetrica e Ginecologica, Università, Torino); M. Mansutti (Azienda Ospedaliera, Udine)

\section{REFERENCES}

1. National Comprehensive Cancer Network (2010) NCCN practice guidelines in oncology. Invasive breast cancer v.3

2. Thürlimann B, Price KN, Gelber RD, et al (2009) Is chemotherapy necessary for premenopausal women with lower-risk node-positive, endocrine responsive breast cancer? 10-year update of International Breast Cancer Study Group Trial 11-93. Breast Cancer Res Treat 113:137-144

3. Pagani O, Gelber S, Simoncini E, et al (2009) Is adjuvant chemotherapy of benefit for postmenopausal women who receive endocrine treatment for highly endocrine-responsive, node-positive breast cancer? International Breast Cancer Study Group Trials VII and 12-93. Breast Cancer Res Treat 116:491-500

4. Albain KS, Barlow WE, Ravdin PM, et al (2009) Adjuvant chemotherapy and timing of tamoxifen in postmenopausal patients with endocrine-responsive, node-positive breast cancer: a phase 3, open-label, randomised controlled trial. Lancet 374:2055-2063

5. Fisher B, Redmond C, Legault-Poisson S, et al (1990) Postoperative chemotherapy and tamoxifen compared with tamoxifen alone in the treatment of positive-node breast cancer patients aged 50 years and older with tumors responsive to tamoxifen: results from the National Surgical Adjuvant Breast and Bowel Project B-16 tral. J Clin Oncol 8:1005-1018 
6. Boccardo F, Rubagotti A, Bruzzi P, et al (1990) Chemotherapy versus tamoxifen versus chemotherapy plus tamoxifen in node-positive, estrogen receptor-positive breast cancer patients: results of a multicentric Italian study. J Clin Oncol 8:1310-1320

7. Rivkin SE, Green S, Metch B, et al (1994) Adjuvant CMFVP versus tamoxifen versus concurrent CMFVP and tamoxifen for postmenopausal, node-positive, and estrogen receptor-positive breast cancer patients: a Southwest Oncology Group study. J Clin Oncol 12:2078-2085

8. International Breast Cancer Study Group (1997) Effectiveness of adjuvant chemotherapy in combination with tamoxifen for node-positive postmenopausal breast cancer patients. J Clin Oncol 15:1385-1394

9. Pritchard KI, Paterson AH, Fine S, et al (1997) Randomized trial of cyclophosphamide, methotrexate, and fluorouracil chemotherapy added to tamoxifen as adjuvant therapy in postmenopausal women with nodepositive ,estrogen and/or progesterone receptor-positive breast cancer: a report of the National Cancer Institute of Canada Clinical Trials Group. J Clin Oncol 15:2302-2311

10. International Breast Cancer Study Group (2006) Tamoxifen after adjuvant chemotherapy for premenopausal women with lymph node-positive breast cancer: International Breast Cancer Study Group Trial 13-93. J Clin Oncol 24:1332-1341

11. Gelber RD, Cole BF, Goldhirsch A, et al (1996) Adjuvant chemotherapy plus tamoxifen compared with tamoxifen alone for postmenopausal breast cancer: meta-analysis of quality adjusted survival. Lancet 347:1066-1071

12. Early Breast Cancer Trialist's Group (2005) Effects of chemotherapy and hormonal therapy for early breast cancer on recurrence and 15-years survival: an overview on the randomised trials. Lancet 365:1687-1717

13. Boccardo F, Rubagotti A, Amoroso D, et al (1992) Chemotherapy versus tamoxifen versus chemotherapy plus tamoxifen in node-positive, oestrogen-receptor positive breast cancer patients. An update at 7 years of the 1st GROCTA (Breast Cancer Adjuvant Chemo-Hormone Therapy Cooperative Group) trial. Eur J Cancer 28:673680

14. Kaplan EL, Meier P (1958) Non parametric estimation from incomplete observation. J Am. Stat. Assoc $53: 457-481$

15. Cox DR. Regression model and life tables (1972). J Royal Stat Soc B 34:187-220

16. Bonetti M., Gelber RD (2000) A graphical method to assess treatment-covariate interactions using the Cox model on subsets of the data. Stat Med 19: 2595-2609

17. Jones MC, Wand MP (1994) Kernel smoothing. Boca Raton, FL. CRC 
18. Gray RJ (1988) A class of k-sample tests for comparing the cumulative incidence of a competing risk. Ann Stat $16: 1141-1154$

19. Pritchard KI (2008) Combining endocrine agents with chemotherapy: which patients and what sequence? Cancer 112:718-722

20. Pico C, Martin M, Jara C, et al (2004) Epirubicin-cyclophosphamide adjuvant chemotherapy plus tamoxifen administered concurrently versus sequentially: randomized phase III trial in postmenopausal node-positive breast cancer patients. A GEICAM 9401 study. Ann Oncol 15:79-87

21. Goldhirsch A, Ingle JN, Gelber RD, et al (2009) Thresholds for therapies: highlights of the St Gallen International Expert Consensus on the primary therapy of early breast cancer 2009. Ann Oncol 20:1319-1329

22. Arpino G, Weiss H, Lee AV, et al (2005) Estrogen receptor-positive, progesterone receptor-negative breast cancer: association with growth factor receptor expression and Tamoxifen resistance. J Natl Cancer Inst $97: 1254-1261$

23. Paik S, Tang G, Shak S, et al (2006) Gene expression and benefit of chemotherapy in women with nodenegative, estrogen receptor-positive breast cancer. J Clin Oncol 24:3726-3734

24. Albain KS, Barlow WE, Shak S, et al (2010) Prognostic and predictive value of the 21-gene recurrence score assay in postmenopausal women with node-positive, oestrogen-receptor-positive breast cancer on chemotherapy: a retrospective analysis of a randomised trial. Lancet Oncol 11:55-65 


\section{Figure-legends}

Fig.1: Kaplan-Meier estimates of Disease-Free Survival; a): all patients; b): pre-menopausal patients; c): postmenopausal patients.

HR= Hazard Ratio; $95 \%$ CI= $95 \%$ Confidence Interval

$\mathrm{CT}=$ Chemotherapy; $\mathrm{T}=$ Tamoxifen $; \mathrm{CTT}=$ Chemotherapy + Tamoxifen

Fig.2: Kaplan-Meier estimates of Overall Survival; a): all patients; b): pre-menopausal patients ;

c): post- menopausal patients.

$\mathrm{HR}=$ Hazard Ratio; $95 \% \mathrm{CI}=95 \%$ Confidence Interval

$\mathrm{CT}=$ Chemotherapy; $\mathrm{T}=$ Tamoxifen $; \mathrm{CTT}=$ Chemotherapy + Tamoxifen

Fig.3: Smoothed hazard rate curves; a): Disease-Free Survival; b): Overall Survival.

$\mathrm{CT}=$ Chemotherapy; $\mathrm{T}=$ Tamoxifen; $\mathrm{CTT}=$ Chemotherapy + Tamoxifen

Fig.4: Cumulative Incidence curves adjusting for Competing Risk

$\mathrm{CT}=$ Chemotherapy $; \mathrm{T}=$ Tamoxifen; $\mathrm{CTT}=$ Chemotherapy + Tamoxifen

Adj = adjusted for: $\mathrm{pT}$, Nodes, menopausal status , PgR concentration $\leq 55 \mathrm{fmol} / \mathrm{mg}$

Fig.5: Stepp showing Cox model Hazard Ratios (T vs. CTT) according to quantitative ER (a: DFS; b: OS) or PgR (c: DFS; d: OS) median tumor concentrations. Horizontal dashed lines: no difference between treatments $(\mathrm{HR}=$ 1,0); horizontal dotted lines: observed treatment difference for the overall population. Solid circles lines: HRs for each-one of the sliding-window sub-populations; bands around these points: simultaneous (across all subgroups) HR 95\% confidence intervals. 
Table 2 Multivariate Analysis of Disease Progression or Mortality: all patients

\begin{tabular}{|c|c|c|c|c|c|c|}
\hline & \multicolumn{3}{|c|}{$\begin{array}{c}\text { Disease } \\
\text { Progression }\end{array}$} & \multicolumn{3}{|c|}{ Mortality } \\
\hline & HR & $(95 \% \mathrm{CI})$ & $\mathbf{p} \leq$ & HR & $(95 \% \mathrm{CI})$ & $\mathrm{p} \leq$ \\
\hline \multicolumn{7}{|c|}{ Treatment } \\
\hline CT & 1 & & 0.004 & 1 & & 0.04 \\
\hline $\mathrm{T}$ & 0.74 & $(0.57-0.98)$ & 0.03 & 0.72 & $(0.56-0.94)$ & 0.015 \\
\hline CTT & 0.64 & $(0.49$ to 0.84$)$ & 0.001 & 0.79 & $(0.61-1.01)$ & 0.06 \\
\hline
\end{tabular}

Menopausal status

Table Ppatient Characteristics 1

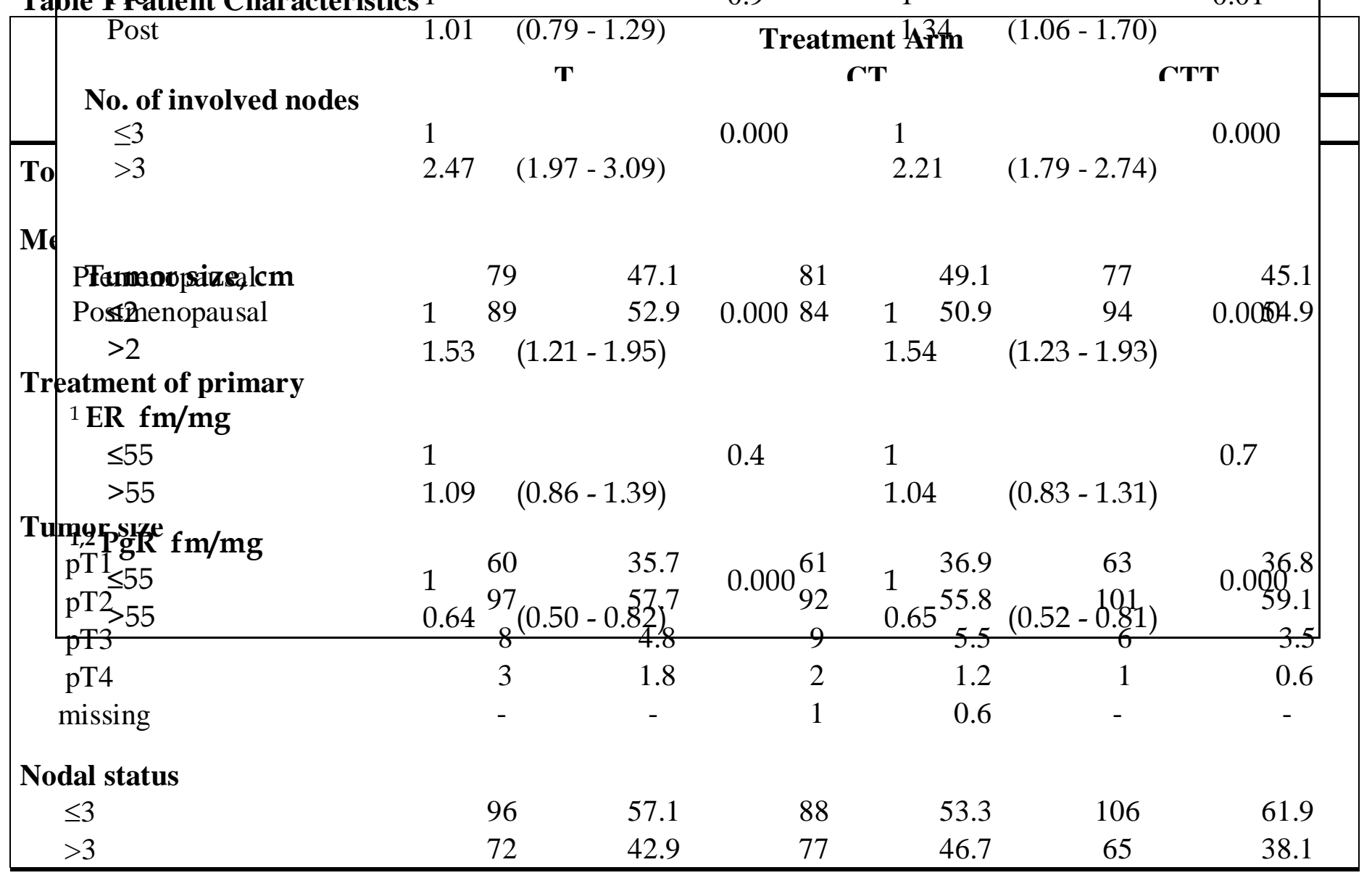

HR $=$ Hazard ratio $;{ }^{1}$ numbers represent median ER or PgR concentration in all patients; ${ }^{2} \mathrm{HRs}$ according to a PgR cutoff level of $10 \mathrm{fm} / \mathrm{mg}$ : $>10$ vs $\leq 10$ : Disease Progression: $0.73(0.55-0.97,95 \%$ CI) $p<0.000$; Mortality: 0.65 (0.48$0.89,95 \% \mathrm{CI}) \mathrm{p}<0.006$ 
Fe to download Figure: DFS Fig. 1A,1B,1C.ppt

Fig. la

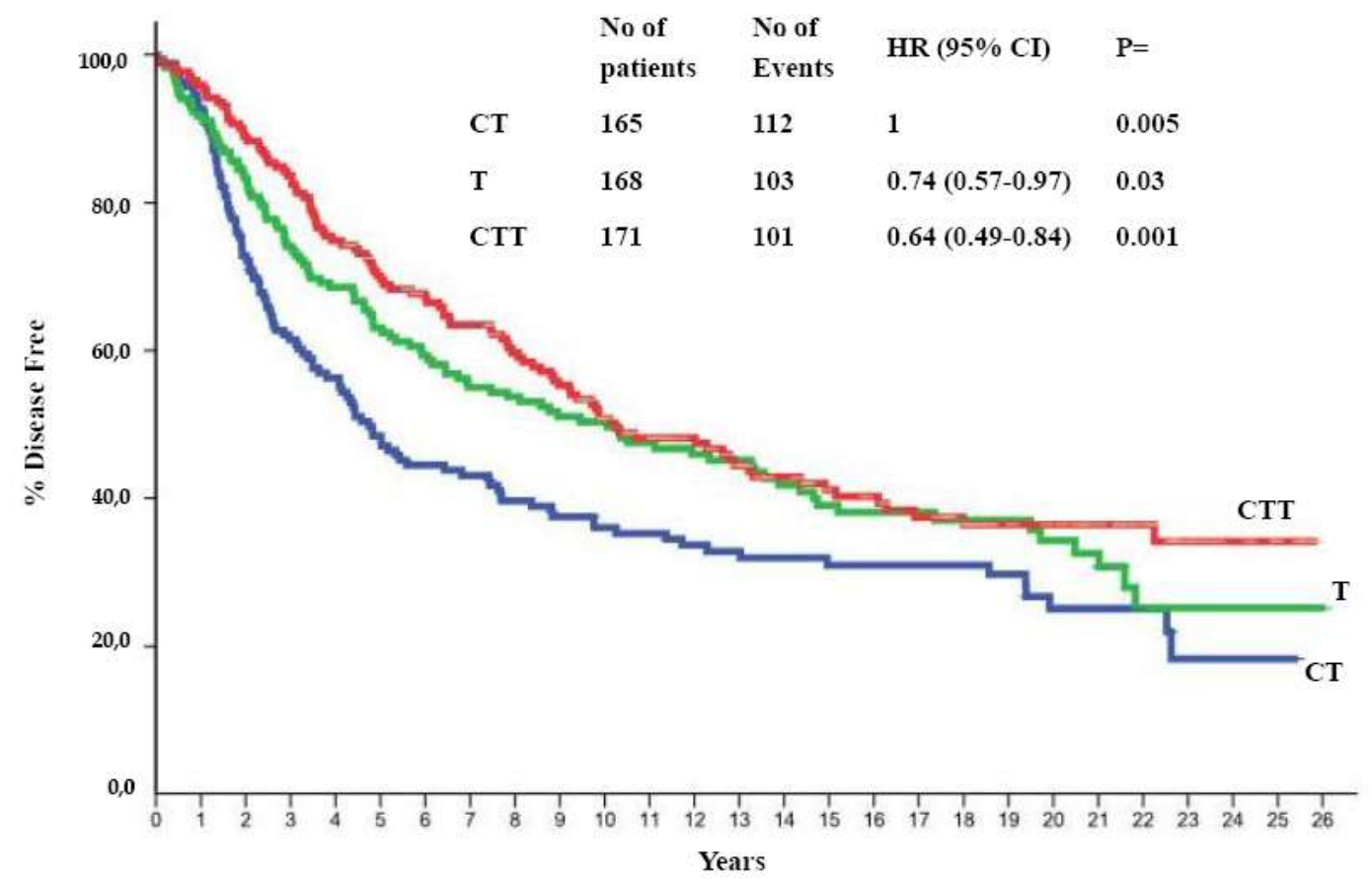


Fig. 1b

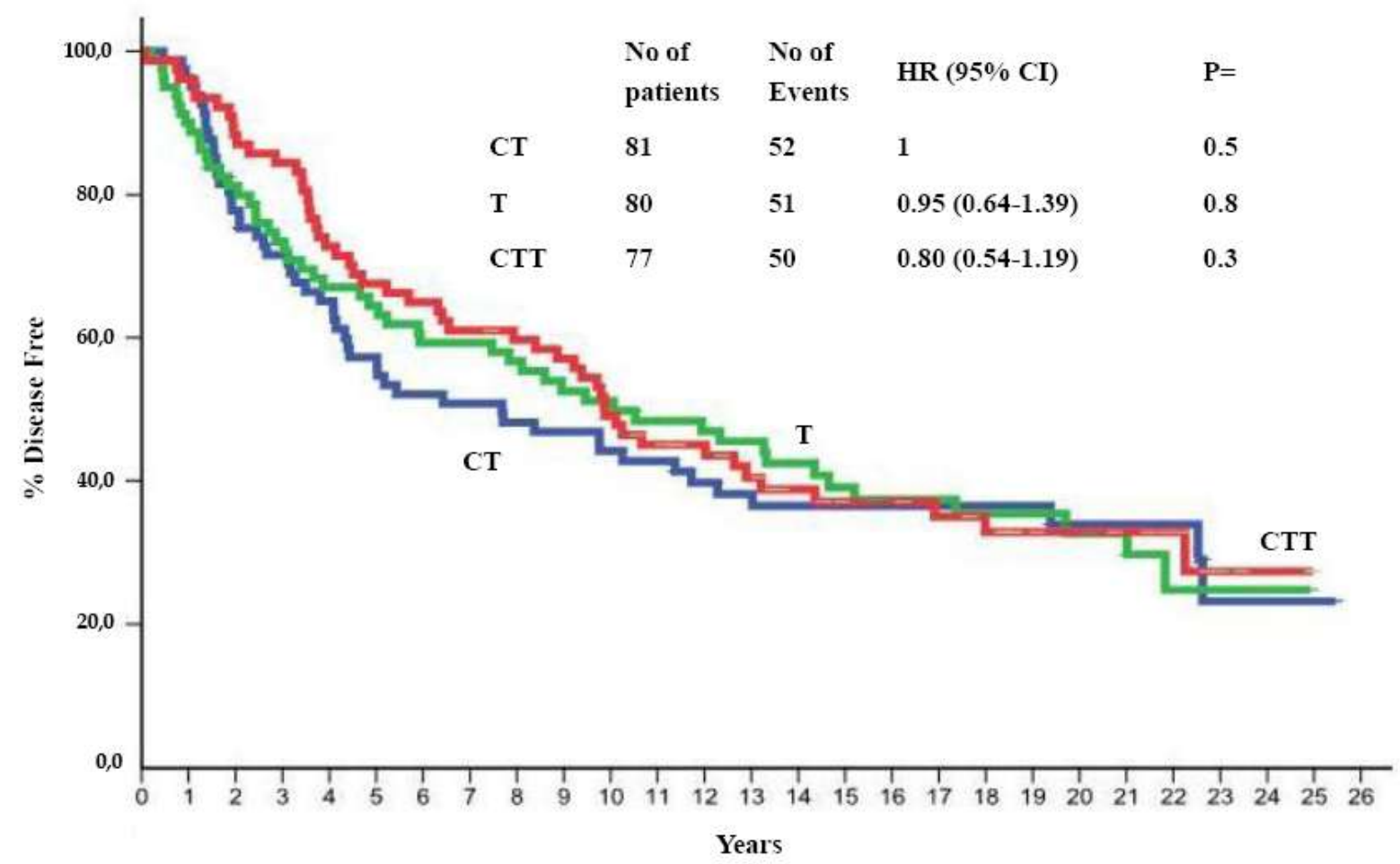

Fig. 1c

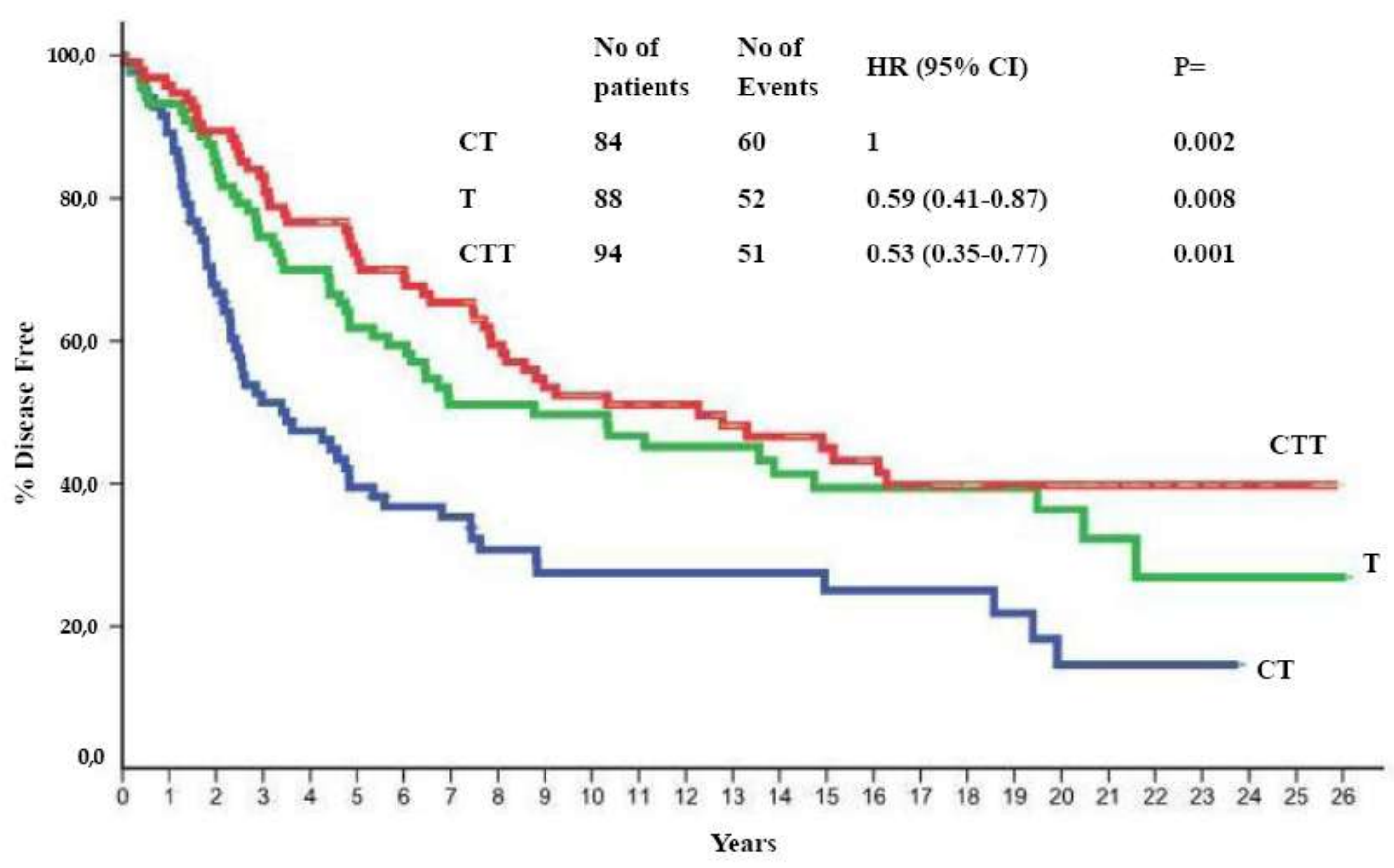




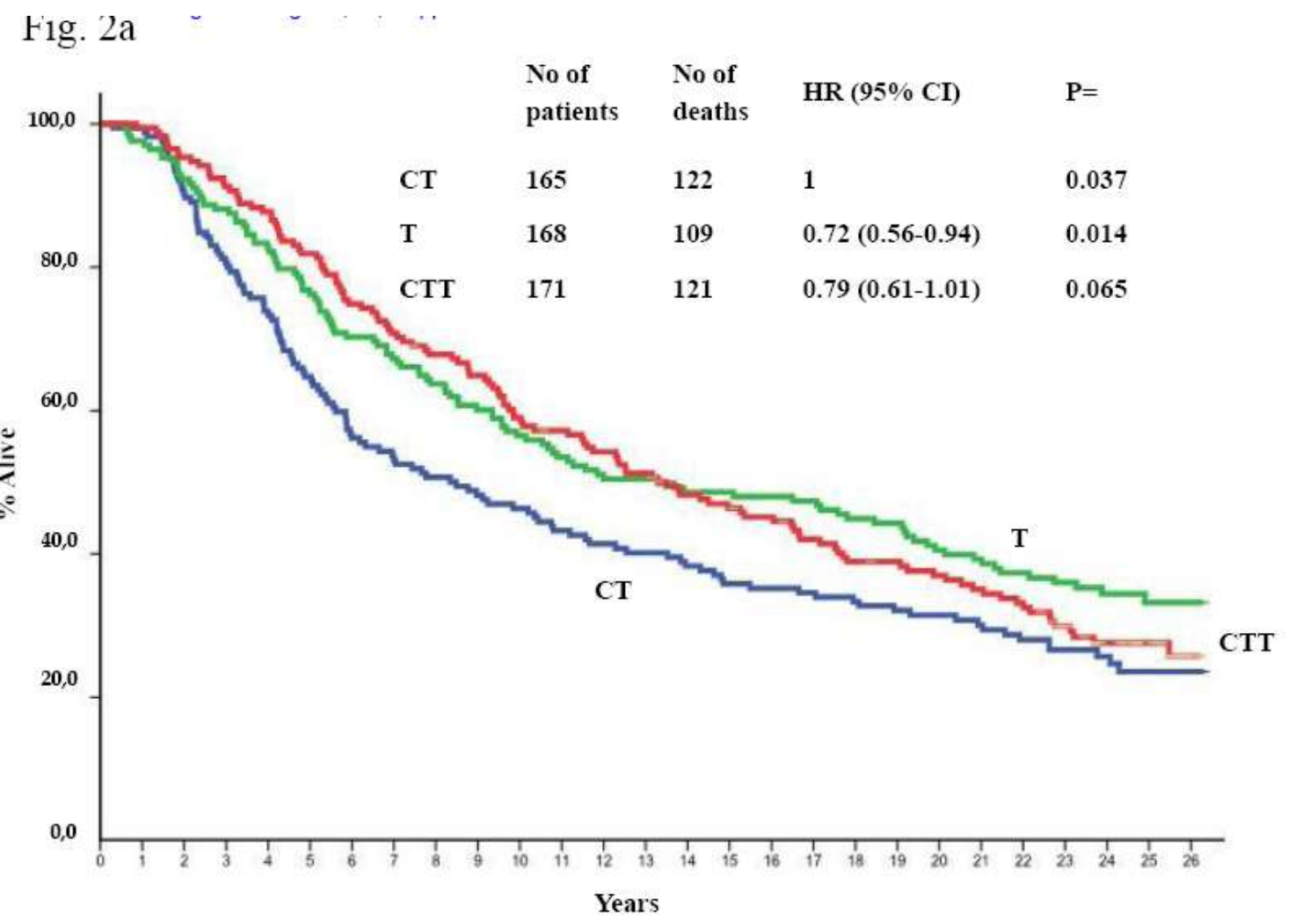


Fig. $2 b$

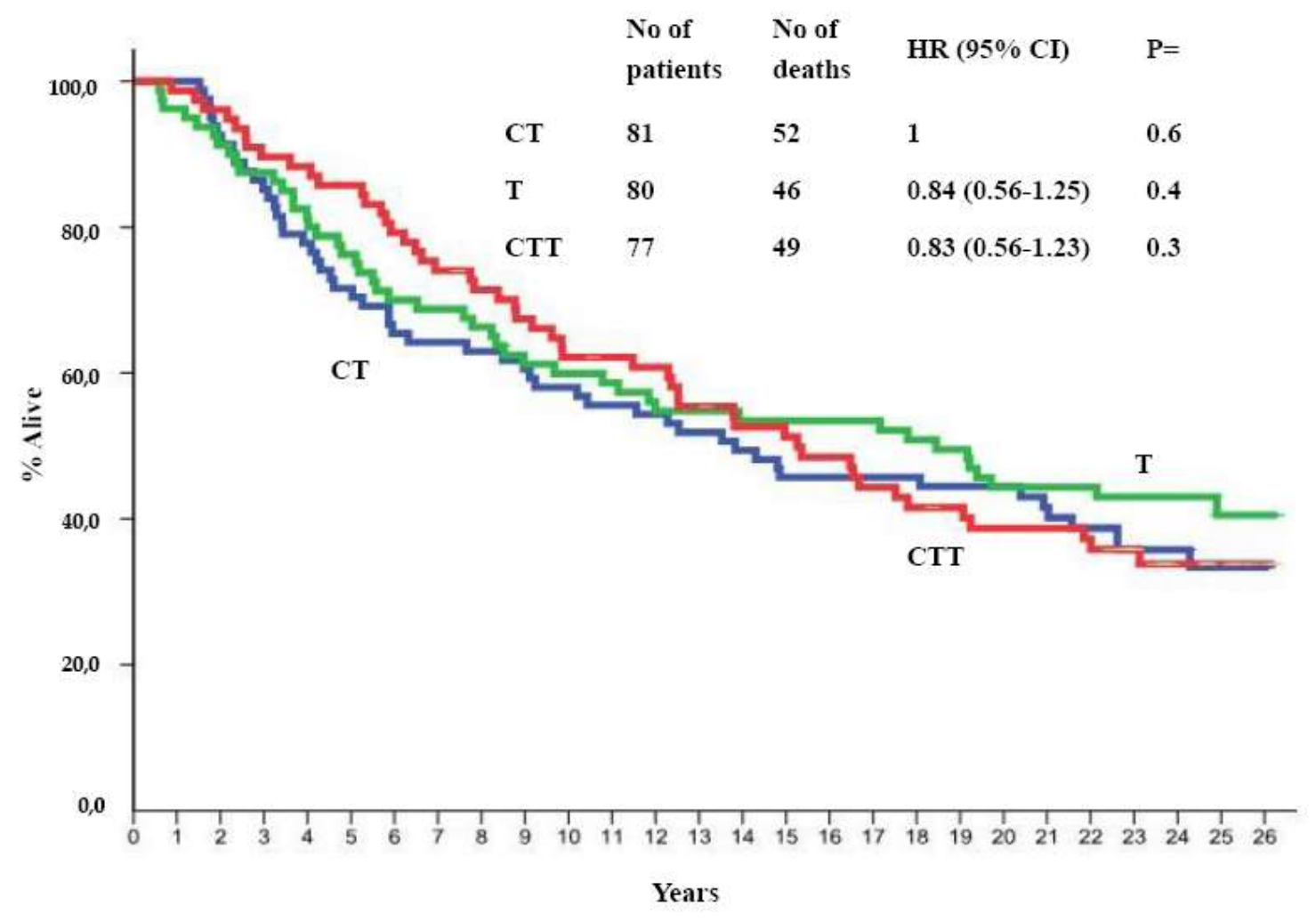




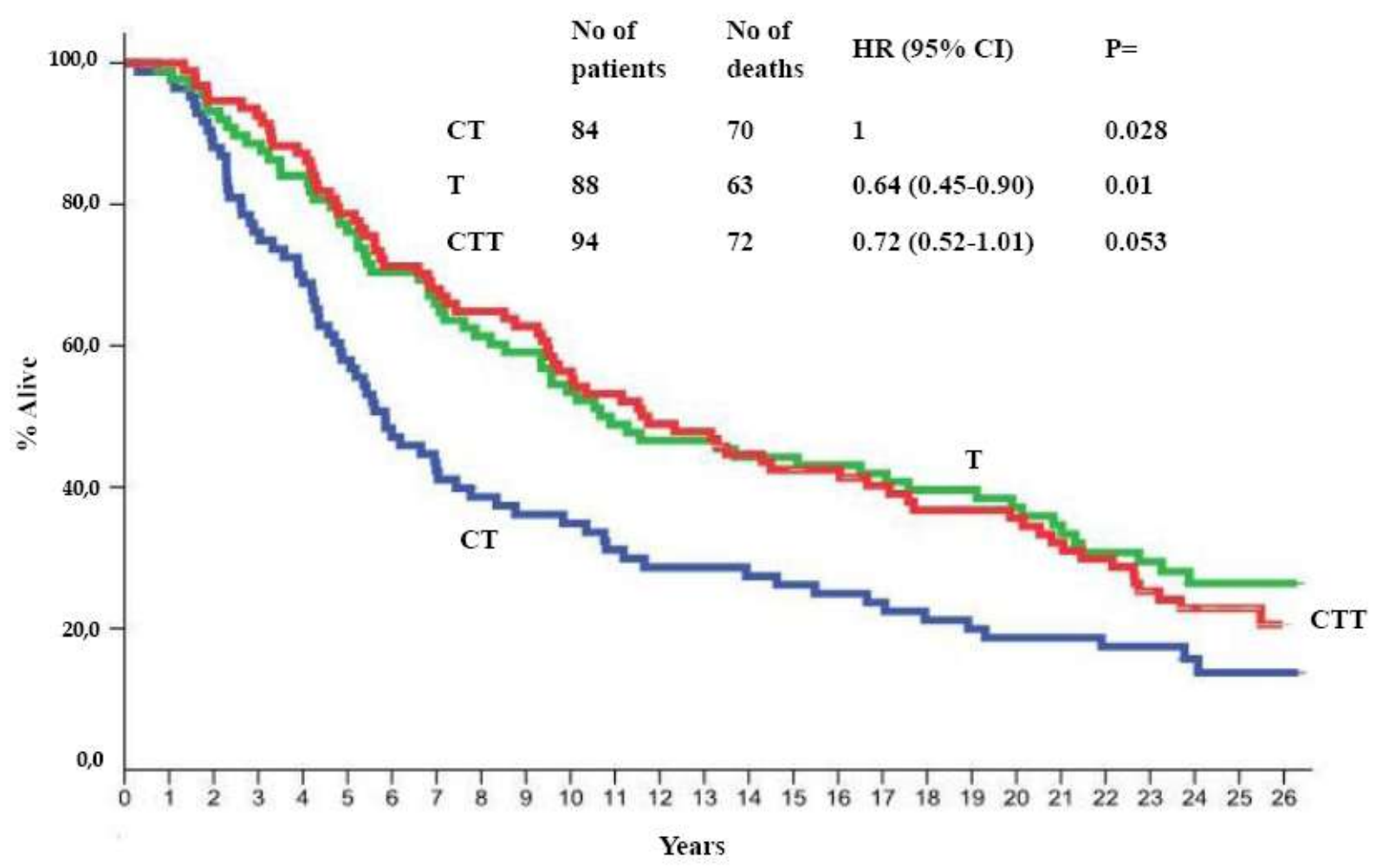

Fig. 3a

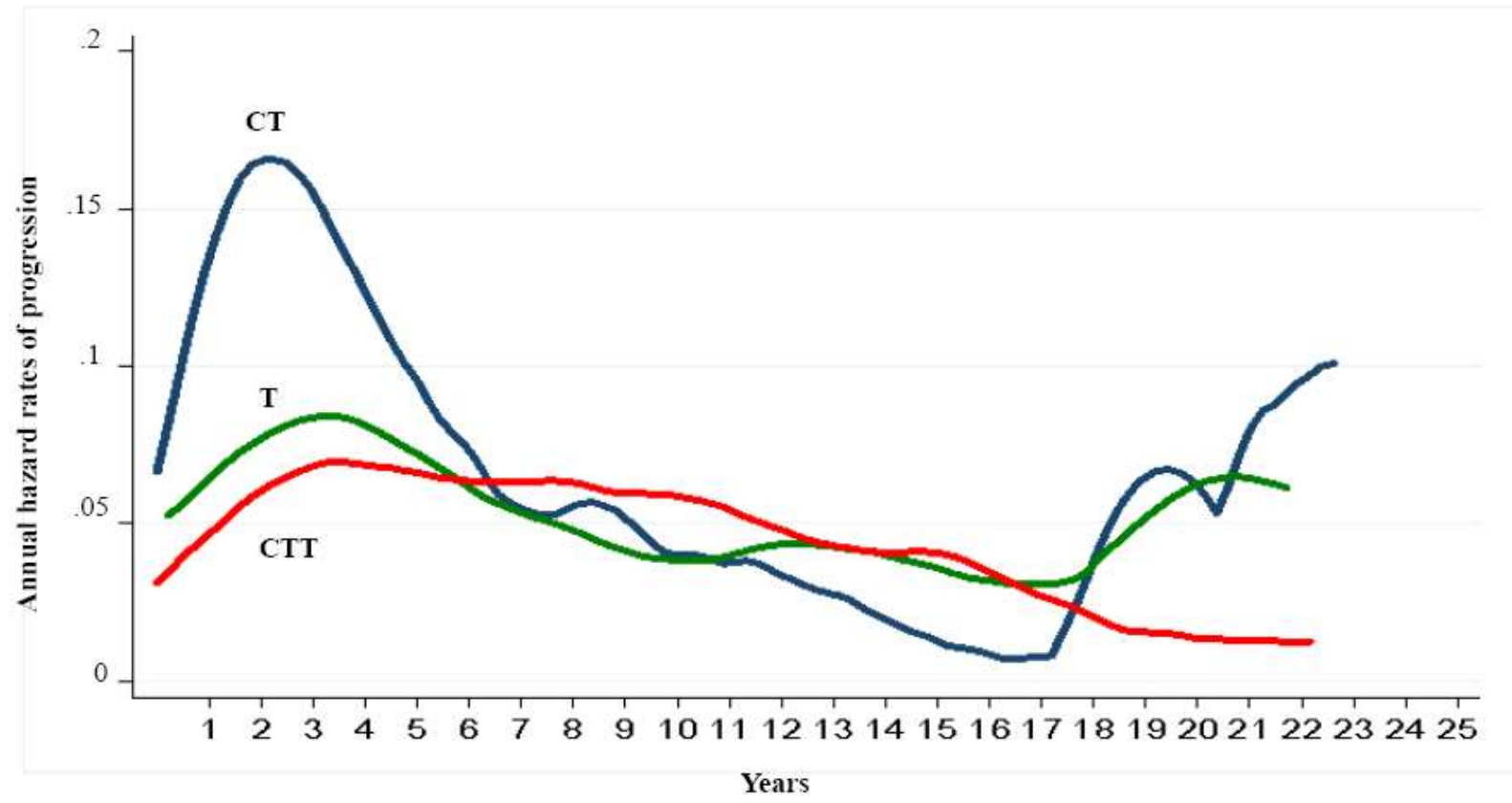


Fig. 3b

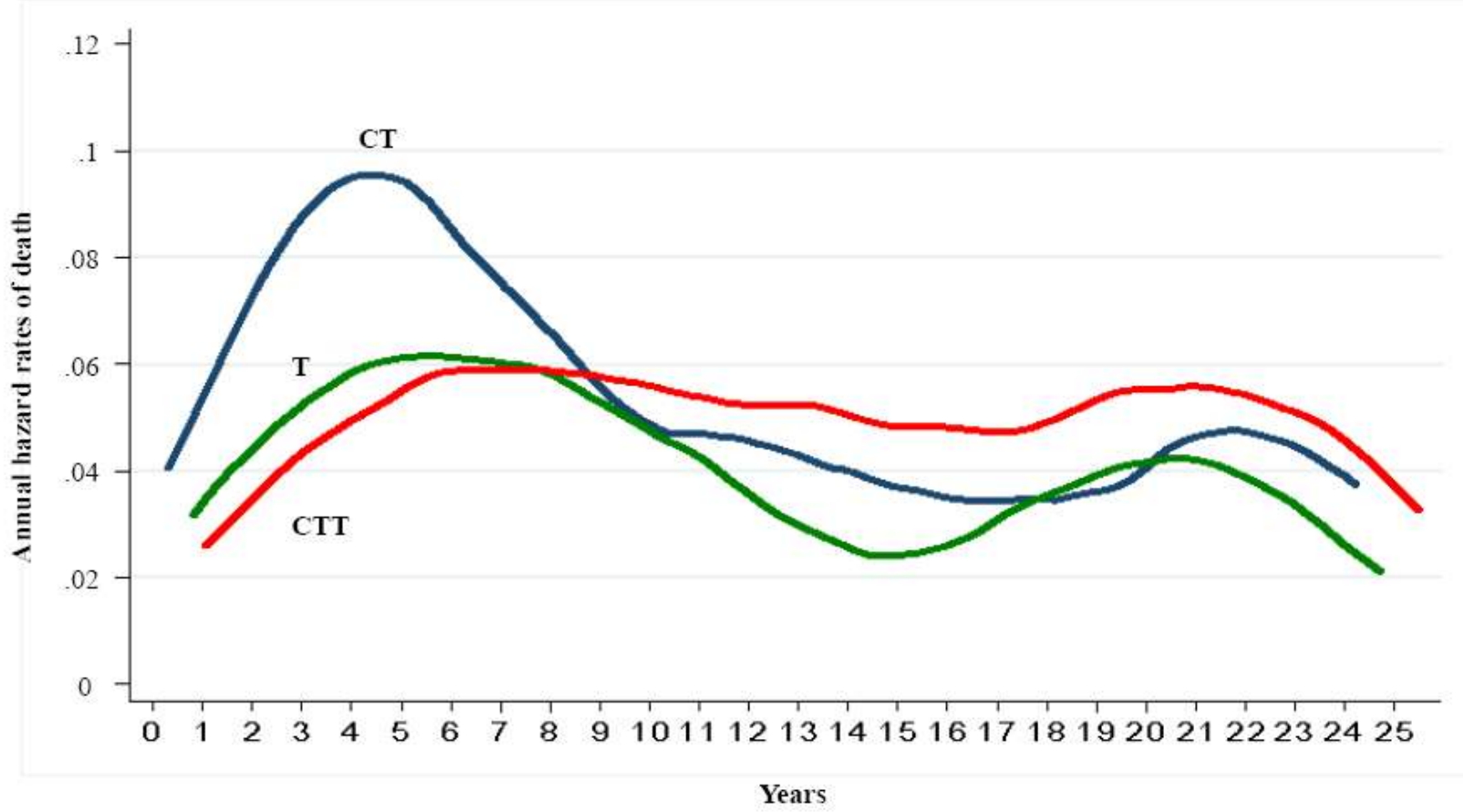

Fig. 4

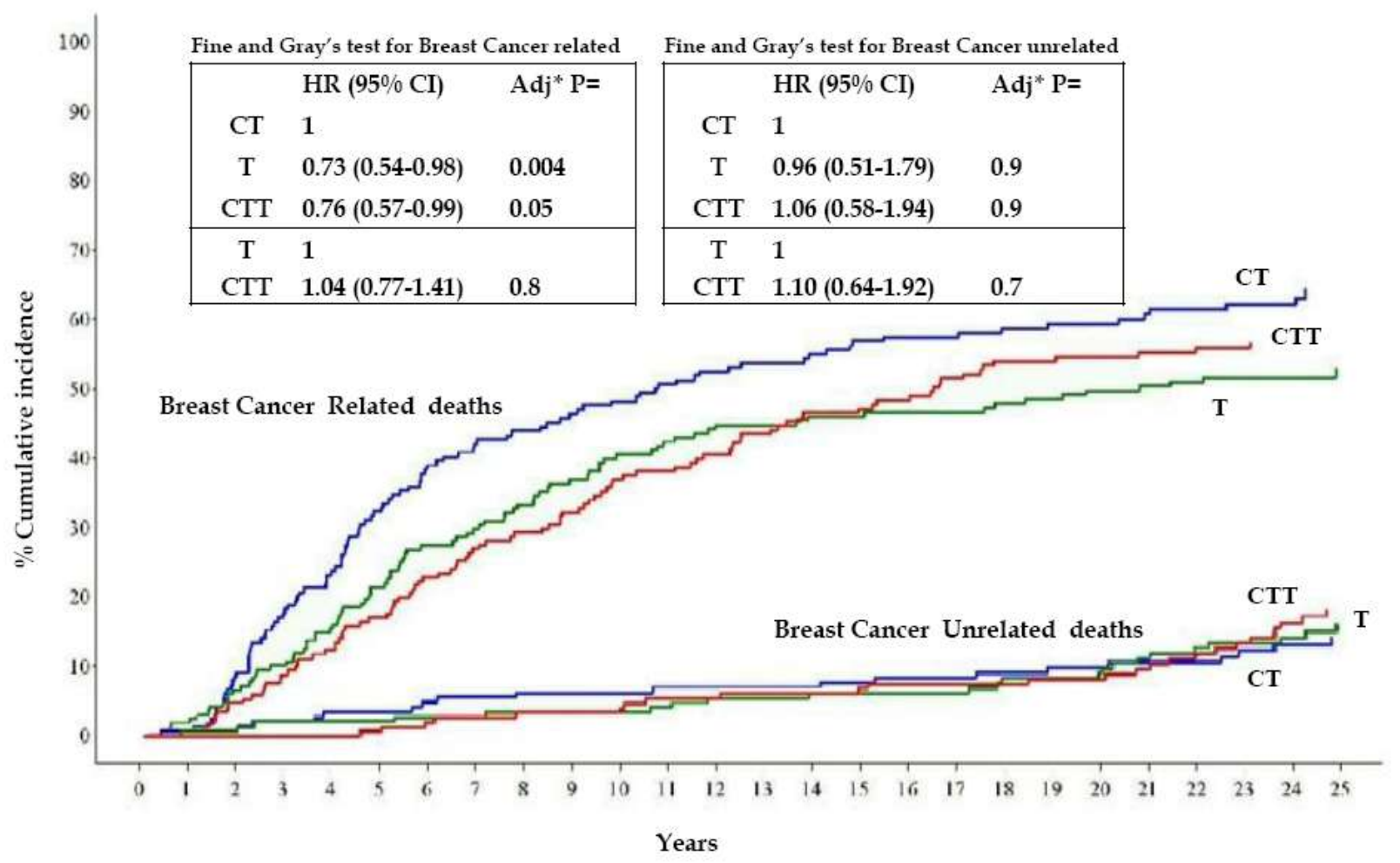


rig. oa

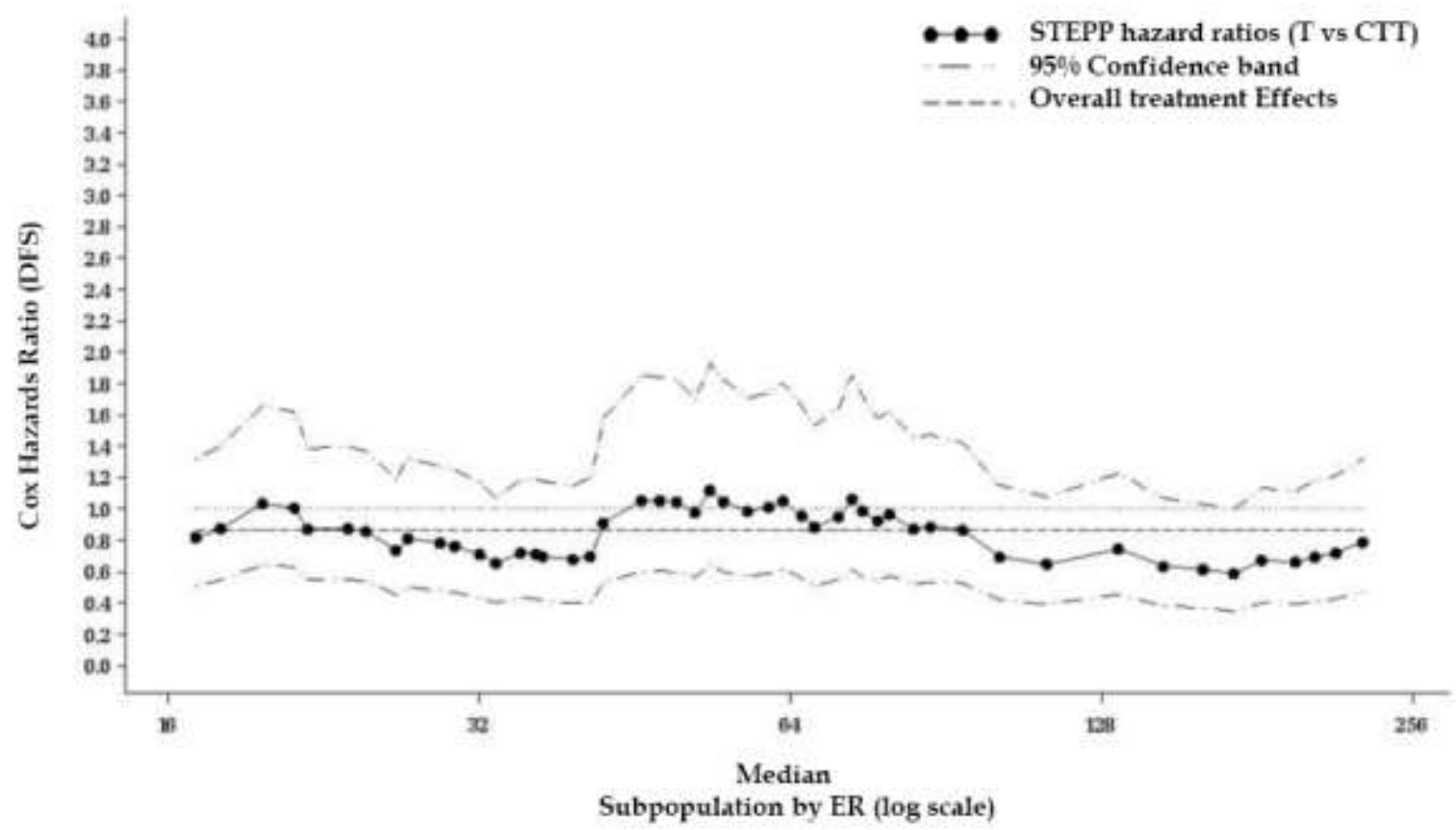

Fig. 5b

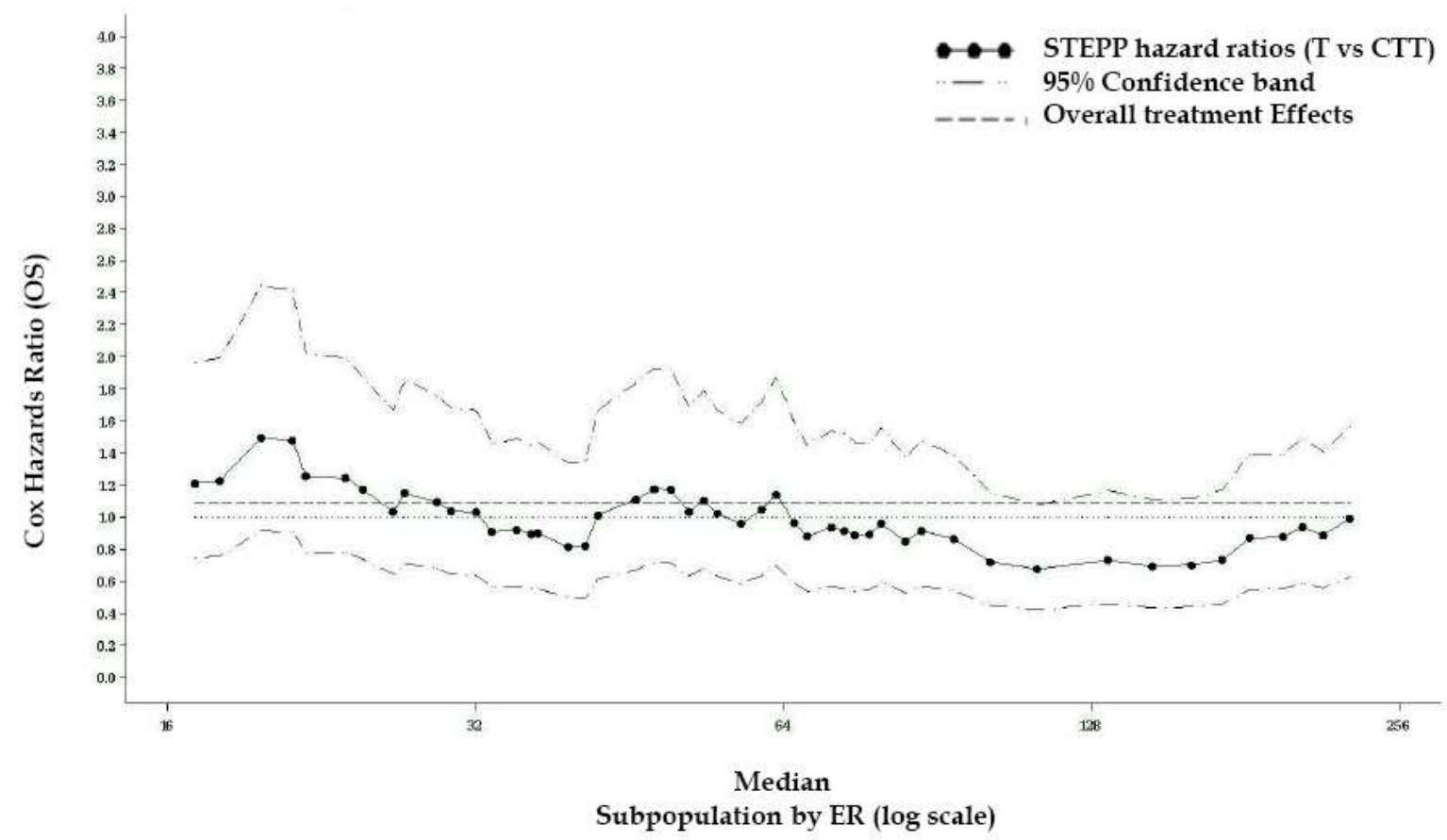


Fig. 5c

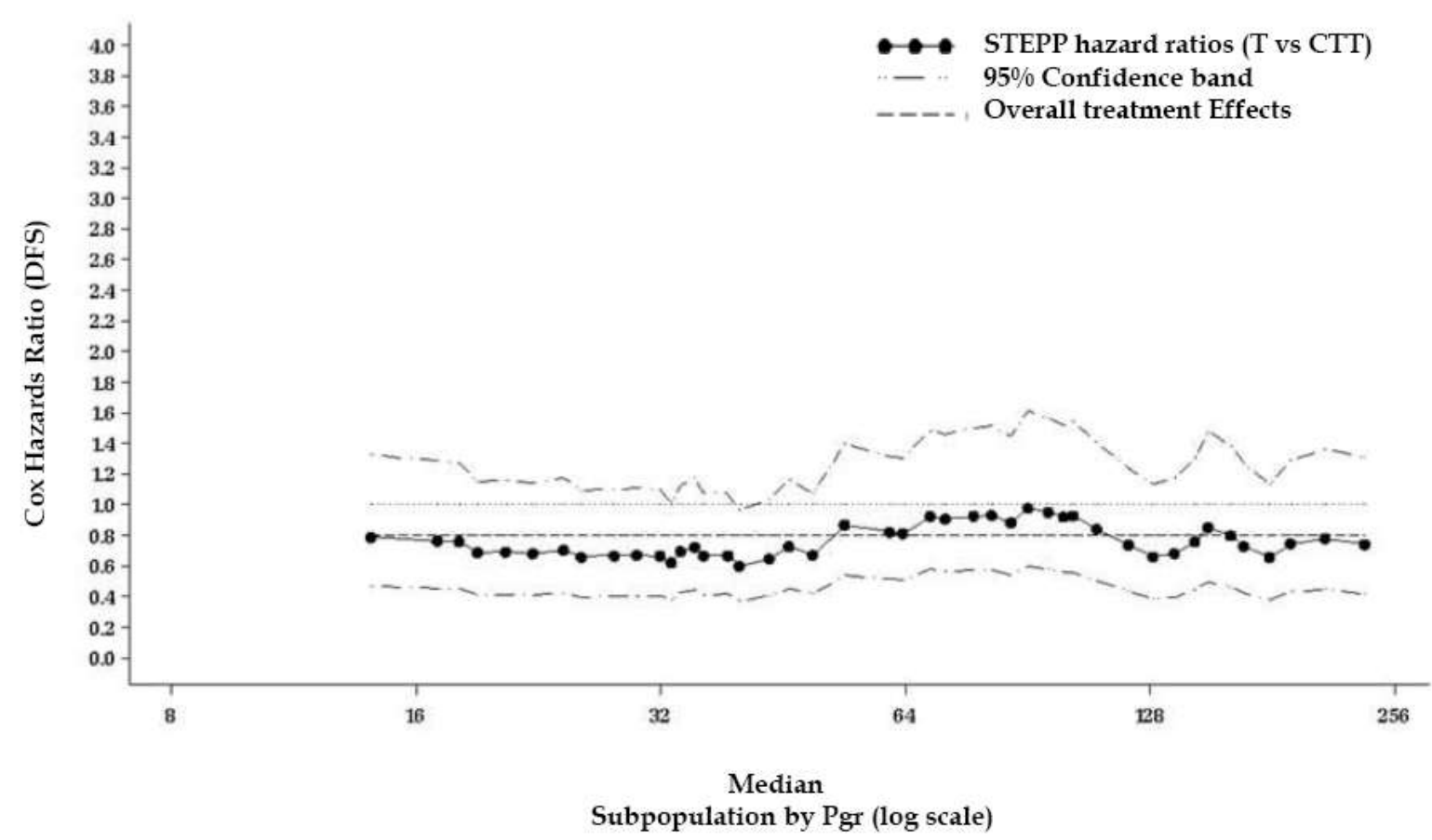

Fig. 5d

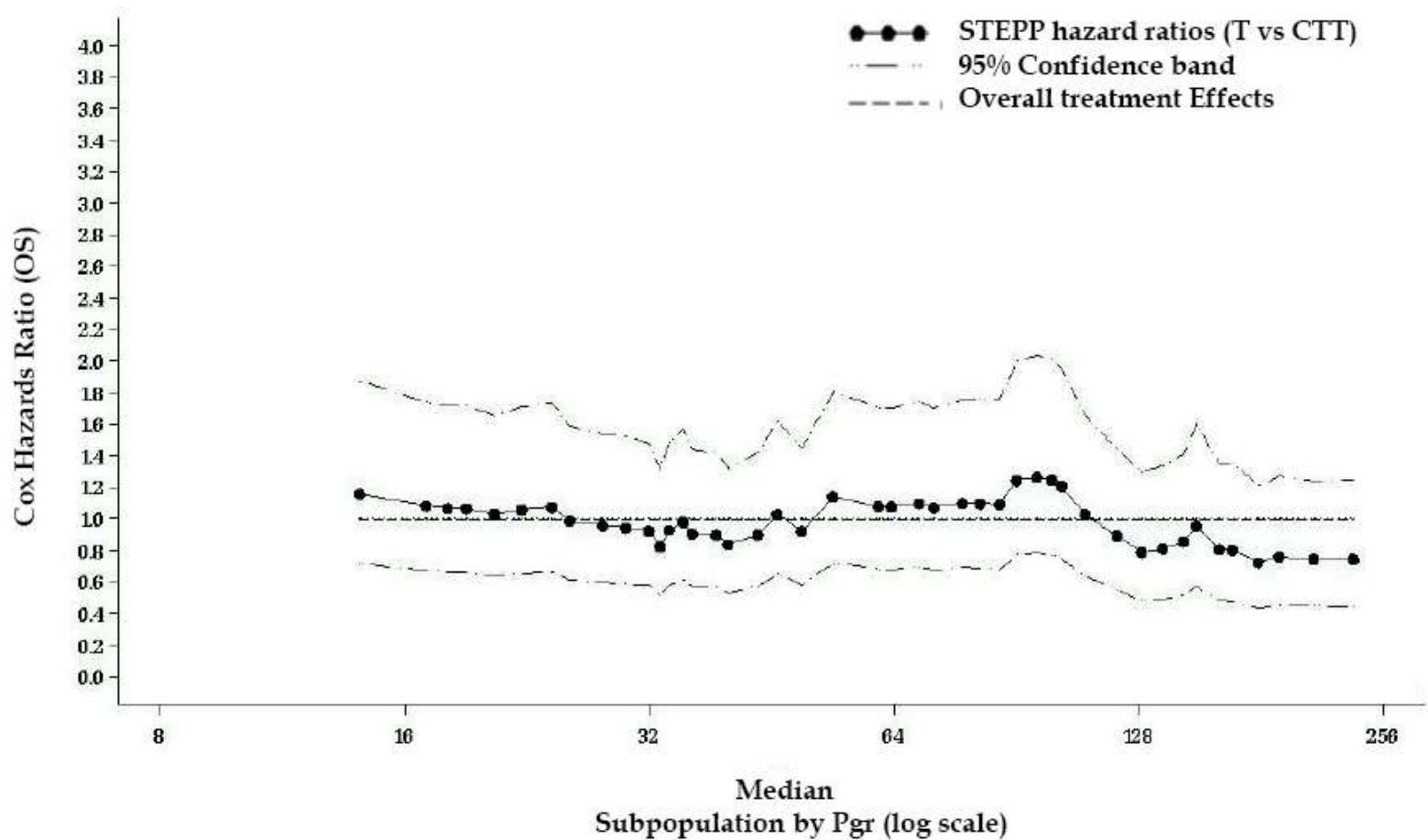


Editorial Manager(tm) for Breast Cancer Research and Treatment Manuscript Draft

Manuscript Number: BREA6490R

Title: Chemotherapy versus Tamoxifen versus Chemotherapy plus Tamoxifen in Node-positive, Estrogen Receptor-positive breast cancer patients. Very late results of the "Gruppo di Ricerca per la Chemio-Ormonoterapia Adiuvante (GROCTA)" 01-Trial in early breast cancer.

Article Type: Clinical trial

Keywords: early breast cancer, chemotherapy, Tamoxifen, chemotherapy plus Tamoxifen

Corresponding Author: Francesco Boccardo, MD

Corresponding Author's Institution: National Cancer Research Institute and University, Genoa

First Author: Francesco Boccardo, MD

Order of Authors: Francesco Boccardo, MD;Pamela Guglielmini, MD;Andrea Parodi, Ph.D;;Alessandra Rubagotti

Abstract: Background and study purposes: firstly, to evaluate whether the benefits of combined chemotherapy (CT) and Tamoxifen (T), previously documented in the GROCTA-01 Trial, were longlasting and, secondly, to show whether ER or PgR levels could allow the identification of patients who could benefit from $T$ alone.

Patients and methods: 504 node-positive, ER-positive, women were randomly assigned to $10 \mathrm{CT}$ courses or to 5 years of T or to the combination of the two (CTT). Disease-free (DFS) and overall survival (OS) were the primary trial-endpoints. DFS data were updated in $75 \%$ of patients and S data in 95\% of them. Cox regression models were used to check for prognostic features, to estimate Hazard Ratios (HRs) for treatment comparisons and to test for possible interaction between variables and treatment effects. Interactions between treatments and ER or PgR median levels were studied with the Sub-population Treatment Effect Pattern Plot (STEPP) methodology.

Results: after a median follow-up time of 21 years, the DFS and os benefits, previously favouring T over CT, continued to be observed, even though they were more evident in the first 6-7 years. The CTT advantages of DFS and OS over T alone were also confirmed. However, the additional benefit was limited to the first 10-12 years as S curves crossed-over afterwards. After STEPP analysis, neither ER or PgR concentrations fully discriminated the patients who could benefit from $\mathrm{T}$ alone. Conclusions: even after such a long follow-up time, we have demonstrated that $\mathrm{T}$ is an effective alternative to $\mathrm{CT}$ for node-positive, ER-positive, breast cancer patients, regardless of their actual menopausal status, and that the additional benefit, especially on late survival, provided by the addition of $\mathrm{CT}$ to this anti-oestrogen, was minimal. 